\title{
EUCLIDEAN TOTALLY DEFINITE QUATERNION FIELDS OVER THE RATIONAL FIELD AND OVER QUADRATIC NUMBER FIELDS
}

\author{
JEAN-PAUL CERRI, JÉRÔME CHAUBERT, AND PIERRE LEZOWSKI
}

\begin{abstract}
In this article we study totally definite quaternion fields over the rational field and over quadratic number fields. We establish a complete list of all such fields which are Euclidean. Moreover, we prove that every field in this list is in fact norm-Euclidean. The proofs are both theoretical and algorithmic.
\end{abstract}

\section{INTRODUCTION}

Quaternion fields are special cases of central division algebras. Let us recall that such an algebra $F$ is a 4 -dimensional algebra over a number field $K$ with basis $(1, i, j, k)$ such that $i^{2}=a, j^{2}=b$ and $k=i j=-j i$, where $a, b$ are non-zero elements of $K$. This algebra is denoted by $\left(\frac{a, b}{K}\right)$. Let $w=x+y i+z j+t k \in\left(\frac{a, b}{K}\right)$, where $x, y, z, t \in K$. We denote by $\bar{w}$ the image of $w$ by the canonical involution of $\left(\frac{a, b}{K}\right)$, which is defined by $\bar{w}=x-y i-z j-t k$, and by $\operatorname{nrd}_{F / K}(w)=w \bar{w}$ its reduced norm. The algebra $\left(\frac{a, b}{K}\right)$ is a division algebra if and only if the quadratic form $\operatorname{nrd}_{F / K}(x+y i+z j+t k)=x^{2}-a y^{2}-b z^{2}+a b t^{2}$ represents zero on $K$ only trivially. In this case, we say that $\left(\frac{a, b}{K}\right)$ is a quaternion field. Throughout this paper, $F$ will be a quaternion field over a number field $K$. We will denote by $\mathbf{Z}_{K}$ the ring of integers of $K$, by $\mathbf{Z}_{K}^{\times}$its unit group and by $N_{K / \mathbf{Q}}$ the norm form. We will also use $N_{K / \mathbf{Q}}$ for the norm of an ideal (if $I$ is a non-zero ideal of $\mathbf{Z}_{K}, N_{K / \mathbf{Q}}(I)=\left|\mathbf{Z}_{K} / I\right|$ ) and $\operatorname{nrd}_{F / K}$ for the reduced norm of an ideal (if $J$ is an ideal of $F, \operatorname{nrd}_{F / K}(J)$ is the ideal of $K$ generated by the $\left.\operatorname{nrd}_{F / K}(x), x \in J\right)$.

Definition 1.1. Let $\Lambda$ be an order of $F$. We say that $\Lambda$ is right-Euclidean if and only if there exist a well-ordered set $W$ and a map $\Phi: \Lambda \longrightarrow W$ such that for every $(a, b) \in \Lambda \times \Lambda \backslash\{0\}$ there exists some $q \in \Lambda$ such that

$$
\Phi(a-b q)<\Phi(b) .
$$

We will also say that $\Phi$ is a right-Euclidean stathm for $\Lambda$.

Let us denote by $N: F \longrightarrow \mathbf{Q}_{\geq 0}$ the absolute value of the reduced norm $\operatorname{map} \operatorname{nrd}_{F / \mathbf{Q}}$ : $F \longrightarrow \mathbf{Q}$ defined by $\operatorname{nrd}_{F / \mathbf{Q}}=N_{K / \mathbf{Q}} \circ \operatorname{nrd}_{F / K}$. The map $N$ is multiplicative and for any order $\Lambda$ of $F$, it satisfies $N(\Lambda) \subseteq \mathbf{Z}_{\geq 0}$. So $N$, with $W=\mathbf{Z}_{\geq 0}$, is a natural and practical candidate for checking whether $\Lambda$ is right-Euclidean, which leads to the following, more precise definition. 
Definition 1.2. An order $\Lambda$ of $F$ is right-norm-Euclidean if for any $(a, b) \in \Lambda \times \Lambda \backslash\{0\}$, there exists some $q \in \Lambda$ such that

$$
N(a-b q)<N(b)
$$

Remark 1.3. We can define similarly left-Euclideanity and left-norm-Euclideanity by replacing bc by cb in (1) and (2). In fact, as we will see in Section 2, these two notions are equivalent, which allows us to speak of Euclidean and norm-Euclidean orders.

Remark 1.4. We will also see that if $F$ admits an Euclidean order $\Lambda$, then $\Lambda$ is necessarily maximal and every maximal order of $F$ is Euclidean. This will enable us to speak of a Euclidean quaternion field without having to specify the order that we consider. This will also be studied in detail in Section 2, where a precise definition of Euclidean quaternion fields will be given.

Unlike the commutative case where the notions described above are applied to number fields, very little is known about Euclidean quaternion fields. For instance, we know the complete list of definite norm-Euclidean quaternion fields over $\mathbf{Q}$ : these are $\left(\frac{-1,-1}{\mathbf{Q}}\right)$, $\left(\frac{-1,-3}{\mathbf{Q}}\right)$ and $\left(\frac{-2,-5}{\mathbf{Q}}\right)$ (see [12] or [5]) and we also know that every indefinite quaternion field over $\mathbf{Q}$ is norm-Euclidean (see [12]). But, except for some rare examples, nothing is known, in terms of complete families, for totally definite quaternion fields over number fields with degree strictly greater than 1 . The aim of this article is to study totally definite Euclidean quaternion fields over quadratic fields and to give their complete list. Our main results are the two following theorems.

Theorem 1.5. The only totally definite Euclidean quaternion fields over a quadratic number field are $\left(\frac{-1,-1}{\mathbf{Q}(\sqrt{2})}\right),\left(\frac{-1,-1}{\mathbf{Q}(\sqrt{5})}\right),\left(\frac{-1,-1}{\mathbf{Q}(\sqrt{13})}\right)$ and $\left(\frac{-1,-3}{\mathbf{Q}(\sqrt{17})}\right)$. Moreover, all of them are norm-Euclidean.

Theorem 1.6. The only (totally) definite Euclidean quaternion fields over $\mathbf{Q}$ are $\left(\frac{-1,-1}{\mathbf{Q}}\right)$, $\left(\frac{-1,-3}{\mathbf{Q}}\right)$ and $\left(\frac{-2,-5}{\mathbf{Q}}\right)$. Moreover, all of them are norm-Euclidean.

The organization of the paper is as follows. In Section 2, we recall some definitions, classical properties and discuss Euclideanity in quaternion fields in general. Then Section 3 is devoted to the proof of Theorem [1.5] in the more specific context of totally definite quaternion fields. The proof is done in two steps. First we prove that the 4 quaternion fields of Theorem 1.5 are norm-Euclidean. Then we establish that the other possible candidates to this property are not Euclidean so that, in particular, they are not norm-Euclidean. Finally, Section 4 shows how the techniques used in Section 3 allow us to obtain Theorem 1.6. When necessary, we have used Magma [1] to compute a maximal order $\Lambda$ and its units modulo $\mathbf{Z}_{K}^{\times}$.

\section{Elementary properties}

In this section, we consider the general case where $F$ is a quaternion field over a number field $K$. 
2.1. Orders and ideals. We first recall some definitions and basic properties. The reader may refer to [6], [10] and [12] for more details. Let $v$ be a place of $K$ and $K_{v}$ be the completion of $K$ at $v$. We say that $v$ is ramified in $F$ if $F_{v}=F \otimes_{K} K_{v}$ is a skew field. An infinite place of $K$ which is ramified in $F$ is necessarily real. The set of places (finite and infinite) which are ramified in $F$ is of even cardinality and uniquely characterizes $F$ up to $K$-algebra isomorphism. If every infinite place of $K$ is ramified in $F$, we say that $F$ is a totally definite quaternion field. As a consequence, if $F$ is a totally definite quaternion field over a number field $K$, then $K$ is necessarily totally real. Moreover, if $K$ is a quadratic field, the number of finite places which are ramified in $F$ is even.

An ideal $I$ of a quaternion field $F$ is a full $\mathbf{Z}_{K}$-lattice in $F$, i.e. such that $K I=F$. An order of $F$ is an ideal which is also a subring of $F$. Equivalently, an order $\Lambda$ of $F$ is a subring of $F$ containing $\mathbf{Z}_{K}$ such that $K \Lambda=F$ and whose elements are integral over $\mathbf{Z}_{K}$. An order is maximal if it is not properly contained in another order. An ideal $I$ defines two orders, its right order and its left order respectively given by: $O_{r}(I)=\{x \in F ; I x \subseteq I\}$ and $O_{l}(I)=\{x \in F ; x I \subseteq I\}$.

Two ideals $I, J$ are left-equivalent if there exists some $x \in F \backslash\{0\}$ such that $I=x J$. The classes of ideals with right-order $\Lambda$ are called the right classes of $\Lambda$. We define in the same way the left classes of $\Lambda$. If $\Lambda$ is a maximal order of $F$, the number of right classes of $\Lambda$ is finite and equal to the number of left classes of $\Lambda$. Moreover this number is independent of the choice of $\Lambda$. It is called the class number of $F$ and we will denote it by $h_{F}$.

Two orders $\Lambda$ and $\Lambda^{\prime}$ of $F$ are of the same type (or conjugate) if there exists some $x \in F \backslash\{0\}$ such that $\Lambda^{\prime}=x^{-1} \Lambda x$. This defines an equivalence relation over the set of maximal orders in $F$. The number of classes for this relation in the set of maximal orders is called the type number of $F$ and we will denote it by $t_{F}$. We have $t_{F} \leq h_{F}$.

An ideal $I$ is two-sided if $O_{r}(I)=O_{l}(I)$, normal if both $O_{r}(I)$ and $O_{l}(I)$ are maximal orders, integral if it is normal and if $I \subseteq O_{r}(I)$. In the latter case, we also have $I \subseteq O_{l}(I)$. For instance, if $\Lambda$ is a maximal order and if $b \in \Lambda \backslash\{0\}$, then $b \Lambda$ is an integral ideal with right order $\Lambda$ and left order its conjugate $b \Lambda b^{-1}$.

If $I$ is integral with right order $\Lambda$, then we have

$$
|\Lambda / I|=N_{K / \mathbf{Q}}\left(\operatorname{nrd}_{F / K}(I)\right)^{2} .
$$

Remark 2.1. As a consequence, if $I$ and $J$ are two integral ideals with right order $\Lambda$ such that $I \subseteq J$ and such that $\operatorname{nrd}_{F / K}(I)=\operatorname{nrd}_{F / K}(J)$, then $I=J$.

Let $\Lambda$ be a maximal order. A prime ideal $\mathfrak{P}$ of $\Lambda$ is a proper integral two-sided ideal with right order $\Lambda$ such that for every pair of two-sided ideals $S$, $T$, with the same properties, if $S T \subseteq \mathfrak{P}$ then $S$ or $T \subseteq \mathfrak{P}$. For every prime ideal $\mathfrak{P}$ of a maximal order $\Lambda$, there exists a unique prime ideal $\mathfrak{p}$ of $\mathbf{Z}_{K}$ such that $\mathfrak{p} \subseteq \mathfrak{P}$ and we have $\mathfrak{p}=\mathfrak{P} \cap \mathbf{Z}_{K}$. Conversely, if $\Lambda$ is a maximal order, for every prime ideal $\mathfrak{p}$ of $\mathbf{Z}_{K}$, there exists a unique prime ideal of $\Lambda$ such that $\mathfrak{p} \subseteq \mathfrak{P}$. With this notation, if the prime $\mathfrak{p}$ is ramified in $F$, then $\mathfrak{p} \Lambda=\mathfrak{P}^{2}$.

A maximal ideal $\mathfrak{N}$ is a maximal element in the set of proper integral ideals with right order $O_{r}(\mathfrak{N})$. In this case, $\mathfrak{N}$ is also maximal in the set of proper integral ideals with left order $O_{l}(\mathfrak{N})$.

For every maximal ideal $\mathfrak{N}$ with right maximal order $\Lambda$, there is a unique prime ideal $\mathfrak{P}$ of $\Lambda$ such that $\mathfrak{P} \subseteq \mathfrak{N}$ and we have $\mathfrak{P}=\{x \in \Lambda ; \Lambda x \subseteq \mathfrak{N}\}$. Then, with the previous notation, we have $\mathfrak{N} \cap \mathbf{Z}_{K}=\mathfrak{P} \cap \mathbf{Z}_{K}=\mathfrak{p}$ and $\operatorname{nrd}_{F / K}(\mathfrak{N})=\mathfrak{p}$. 
A proper product of ideals is a product $N_{1} \cdots N_{l}$ where for every $1 \leq i \leq l-1, \mathcal{O}_{r}\left(N_{i}\right)=$ $\mathcal{O}_{l}\left(N_{i+1}\right)$. Every proper integral ideal $I$ admits a decomposition into a proper product of maximal ideals $I=\mathfrak{N}_{1} \cdots \mathfrak{N}_{l}$ where $O_{l}(I)=O_{l}\left(\mathfrak{N}_{1}\right)$ and $O_{r}(I)=O_{r}\left(\mathfrak{N}_{l}\right)$ (see [10]).

Lemma 2.2. We have the following properties.

(i) With the above notation, we have $\operatorname{nrd}_{F / K}(I)=\operatorname{nrd}_{F / K}\left(\mathfrak{N}_{1}\right) \cdots \operatorname{nrd}_{F / K}\left(\mathfrak{N}_{l}\right)$.

(ii) If $\mathfrak{p}$ is a prime ideal of $\mathbf{Z}_{K}$ which is ramified in $F$, there exists a unique maximal ideal $\mathfrak{N}$ of $F$ such that $\mathfrak{p} \subseteq \mathfrak{N}$. Moreover, $\mathfrak{N}$ is two-sided.

(iii) Suppose that $h_{F}=1$. Let $\mathfrak{p}$ be a prime ideal of $\mathbf{Z}_{K}$ which is ramified in $F$ and let $\mathfrak{N}$ be the unique maximal ideal of $F$ as defined in (ii), with right order $\Lambda$. Let $x, y \in \Lambda$ such that $x y \in \mathfrak{N}$. Then $x$ or $y \in \mathfrak{N}$.

(iv) Under the hypotheses of (iii), suppose that $x \in \Lambda$ satisfies $\operatorname{nrd}_{F / K}(x) \in \mathfrak{p}$. Then $x \in \mathfrak{N}$. Moreover, if $\operatorname{nrd}_{F / K}(x) \mathbf{Z}_{K}=\mathfrak{p}$, we have $x \Lambda=\mathfrak{N}$.

Proof. (i) We can prove this property by induction on $l$ where $\mathfrak{N}_{1} \cdots \mathfrak{N}_{l}$ is a proper product of ideals such that $O_{r}\left(\mathfrak{N}_{i}\right)$ is maximal for every $1 \leq i \leq l-1$ (which will be the case if the $\mathfrak{N}_{i}$ are normal, and in particular maximal). In fact the property holds for $l=1$ (trivial) and $l=2$ since $O_{r}\left(\mathfrak{N}_{1}\right)=O_{l}\left(\mathfrak{N}_{2}\right)$ (see [12]). Suppose that it is true for $l-1 \geq 2$. Put $\mathfrak{N}_{2}^{\prime}=\mathfrak{N}_{1} \mathfrak{N}_{2}$. We have $O_{r}\left(\mathfrak{N}_{2}\right) \subseteq O_{r}\left(\mathfrak{N}_{2}^{\prime}\right)$, but $O_{r}\left(\mathfrak{N}_{2}\right)$ is maximal and in fact, we have $O_{r}\left(\mathfrak{N}_{2}^{\prime}\right)=O_{r}\left(\mathfrak{N}_{2}\right)=O_{l}\left(\mathfrak{N}_{3}\right)$. Then we use the induction hypothesis with $\mathfrak{N}_{2}^{\prime}$ and the $\mathfrak{N}_{i}$, $i \geq 3$.

(ii) Suppose that $\mathfrak{N}$ is a maximal ideal such that $\mathfrak{p} \subseteq \mathfrak{N}$. Let $\mathfrak{P}$ be the unique two-sided ideal above $\mathfrak{p}$. We have $\mathfrak{P} \subseteq \mathfrak{N}$. Moreover $\operatorname{nrd}_{F / K}(\mathfrak{N})=\mathfrak{p}$ and from $\mathfrak{p} \Lambda=\mathfrak{P}^{2}$, we have $\operatorname{nrd}_{F / K}(\mathfrak{P})=\mathfrak{p}$. Remark 2.1 shows that necessarily $\mathfrak{N}=\mathfrak{P}$.

(iii) Suppose that $y \notin \mathfrak{N}$. Then $I=\mathfrak{N}+y \Lambda$ is an ideal with right order $\Lambda$ strictly containing $\mathfrak{N}$. Moreover, since $h_{F}=1$, there exists some $b \in \Lambda \backslash\{0\}$ such that $I=b \Lambda$. This implies that $I$ is an integral ideal and by maximality of $\mathfrak{N}, I$ is necessarily equal to $\Lambda$. Hence, there exist $a \in \mathfrak{N}$ and $\lambda \in \Lambda$ such that $a+y \lambda=1$. This implies that $x a+x y \lambda=x$. As $\mathfrak{N}$ is two-sided, $x a$ and $x y \lambda \in \mathfrak{N}$, so that $x \in \mathfrak{N}$.

(iv) If $\operatorname{nrd}_{F / K}(x) \in \mathfrak{p}$, then $x \bar{x} \in \mathfrak{N}$ and by (iii), $x$ or $\bar{x} \in \mathfrak{N}$. In the latter case $x \in \overline{\mathfrak{N}}$, but $\overline{\mathfrak{N}}$ is also a maximal integral ideal above $\mathfrak{p}$ with left order $\bar{\Lambda}$ and right order $\overline{\Lambda^{\prime}}$, where $\Lambda^{\prime}$ is the left order of $\mathfrak{N}$. In fact, as we will see in Lemma 2.5, these orders are respectively $\Lambda$ and $\Lambda^{\prime}$. Since $\mathfrak{N}$ is the unique maximal ideal above $\mathfrak{p}$ by (ii), we have $\overline{\mathfrak{N}}=\mathfrak{N}$ and $x \in \mathfrak{N}$. Moreover if $\operatorname{nrd}_{F / K}(x) \mathbf{Z}_{K}=\mathfrak{p}$, we have $\operatorname{nrd}_{F / K}(\mathfrak{N})=\mathfrak{p}=\operatorname{nrd}_{F / K}(x \Lambda)$. As $x \Lambda \subseteq \mathfrak{N}$, Remark 2.1 gives us the result.

2.2. The transfinite construction. Now, let us follow the approach of Motzkin [9] and Samuel [11] for the commutative cased. Suppose that $\Lambda$ is right-Euclidean for some stathm $\Phi: \Lambda \longrightarrow W$. Let us denote by $E$ the non-empty set of right-Euclidean stathms for $\Lambda$ taking their values in $W$. It is easy to see that $\Lambda$ is right-Euclidean for $\Psi=\inf _{\phi \in E} \phi$, which is consequently the smallest right-Euclidean stathm for $\Lambda$ (with respect to $W$ ). Let us notice that $\Psi(x)=0 \Longleftrightarrow x=0$, and that if $\lambda=\min \{\Psi(x) ; x \in \Lambda \backslash\{0\}\}$, the set $\{x \in \Lambda ; \Psi(x)=\lambda\}$ is $\Lambda^{\times}$, the set of units of $\Lambda$.

\footnotetext{
${ }^{1}$ Samuel does not use the commutativity property of the rings and the only change to do is to write $b \Lambda$ instead of $A a$ because we are working with right-Euclideanity.
} 
To simplify, let us first remark that, as in the ring case, we can take $W=\mathbf{Z}_{\geq 0}$. This is possible because for any non-zero $b \in \Lambda$, the set $\Lambda / b \Lambda$ is finite (see [11, Proposition 15]). Now, for $n \in \mathbf{Z}_{\geq 0}$, let us put

$$
\Lambda_{n}=\{\lambda \in \Lambda ; \Psi(\lambda) \leq n\} .
$$

For instance, we have $\Lambda_{0}=\{0\}, \Lambda_{1}=\{0\} \cup \Lambda^{\times}$. We can now prove

Theorem 2.3. For every $n \in \mathbf{Z}_{\geq 1}$ we have

$$
\Lambda_{n}=\{0\} \cup\left\{b \in \Lambda \backslash\{0\} \text { s.t. the canonical map } \Lambda_{n-1} \longrightarrow \Lambda / b \Lambda \text { is onto }\right\} .
$$

Proof. The proof is the same as in the commutative case. See [5] for details.

This leads to the following transfinite construction and criterion.

Corollary 2.4. We put $\Lambda_{0}=\{0\}$ and for $n \geq 1$ we define $\Lambda_{n}$ by induction as in (31). Then the sequence $\left(\Lambda_{n}\right)_{n \geq 0}$ is increasing and $\Lambda$ is right-Euclidean if and only if

$$
\bigcup_{n \geq 0} \Lambda_{n}=\Lambda
$$

Moreover if $\Psi$ is the smallest right-Euclidean stathm for $\Lambda$, for $n>0$ and $\lambda \in \Lambda$, we have

$$
\Psi(\lambda)=n \Longleftrightarrow \lambda \in \Lambda_{n} \backslash \Lambda_{n-1} .
$$

Proof. Elementary (see [5]).

\subsection{Initial remarks.}

Lemma 2.5. Let $\Lambda$ be an order of $F$. Then $\bar{\Lambda}=\Lambda$.

Proof. Let $\lambda \in \Lambda$. Let us denote by $\operatorname{Tr}_{F / K}$ the trace map defined by $\operatorname{Tr}_{F / K}(x)=x+\bar{x}$. Since $\lambda$ is in an order of $F$ we have $\operatorname{Tr}_{F / K}(\lambda) \in \mathbf{Z}_{K} \subseteq \Lambda$, from which we deduce $\bar{\lambda}=\operatorname{Tr}_{F / K}(\lambda)-\lambda \in \Lambda$. This proves that $\bar{\Lambda} \subseteq \Lambda$ and by symmetry we have an equality.

Proposition 2.6. An order $\Lambda$ of $F$ is right-Euclidean if and only if it is left-Euclidean.

Proof. Suppose that $\Lambda$ is right-Euclidean, equipped with a stathm $\Phi: \Lambda \longrightarrow \mathbf{Z}_{\geq 0}$ (for instance its minimal Euclidean stathm). Since $\bar{\Lambda}=\Lambda$, we can define a map $\Phi^{\prime}: \Lambda \longrightarrow \mathbf{Z}_{\geq 0}$ by $\Phi^{\prime}(\lambda)=\Phi(\bar{\lambda})$. Let $a, b \in \Lambda$ with $b \neq 0$. Since $\bar{a}, \bar{b} \in \Lambda$ and $\bar{b} \neq 0$, there exists some $q \in \Lambda$ such that $\Phi(\bar{a}-\bar{b} q)<\Phi(\bar{b})$. This implies that $\Phi^{\prime}(a-\bar{q} b)<\Phi^{\prime}(b)$. Since $\bar{q} \in \Lambda$, we see that $\Lambda$ equipped with $\Phi^{\prime}$ is left-Euclidean, and we can conclude by symmetry.

This leads to

Definition 2.7. An order of $F$ will be said to be Euclidean if it is left or right-Euclidean.

Proposition 2.8. Let $\Lambda$ be a Euclidean order of $F$. Then $\Lambda$ is necessarily maximal.

Proof. Let us equip $\Lambda$ with a right-Euclidean stathm $\Phi$ and let us consider $\Lambda^{\prime}$ a maximal order containing $\Lambda$. We want to prove that $\Lambda=\Lambda^{\prime}$. The set

$$
E=\left\{a \in \Lambda \backslash\{0\} ; a \Lambda^{\prime} \subseteq \Lambda\right\}
$$

is non-empty. Let $\alpha \in E$ such that

$$
\Phi(\alpha)=\inf \{\Phi(a) ; a \in E\} .
$$


For any $\lambda^{\prime} \in \Lambda^{\prime}$, we have $\alpha \lambda^{\prime} \in \Lambda, \alpha \in \Lambda \backslash\{0\}$ and there exists a $\lambda \in \Lambda$ such that

$$
\Phi\left(\alpha \lambda^{\prime}-\alpha \lambda\right)<\Phi(\alpha) .
$$

But

$$
\left(\alpha \lambda^{\prime}-\alpha \lambda\right) \Lambda^{\prime} \subseteq \alpha \Lambda^{\prime} \subseteq \Lambda .
$$

Moreover we have $\alpha \lambda^{\prime}-\alpha \lambda \in \Lambda$. Consequently $\alpha \lambda^{\prime}-\alpha \lambda \in E \cup\{0\}$, and (4) implies $\alpha \lambda^{\prime}-\alpha \lambda=0$, by choice of $\alpha$. This leads to $\lambda^{\prime}=\lambda \in \Lambda$, from which we deduce that $\Lambda^{\prime} \subseteq \Lambda$.

Now, as in the number field case, Euclideanity implies principality.

Proposition 2.9. If $F$ admits a Euclidean (necessarily maximal) order $\Lambda$, then $h_{F}=1$.

Proof. The proof is the same as in the commutative case. It is sufficient to prove for instance that every ideal $I$ with right order $\Lambda$ (equipped with $\Phi$ ) is principal. Up to equivalence we may assume that $I \subseteq \Lambda$. Let us take $b \in I$ such that $\Phi(b)=\min \{\Phi(x) ; x \in I \backslash\{0\}\}$. We have $b \Lambda \subseteq I$. Now, if $a \in I$ there exists some $q \in \Lambda$ such that $\Phi(a-b q)<\Phi(b)$. But $a-b q \in I$, which implies $a=b q$ by choice of $b$. Finally $I=b \Lambda$.

Proposition 2.10. If $F$ admits a Euclidean maximal order, then every maximal order is also Euclidean.

Proof. Since $F$ has class number one, we have $t_{F}=1$ and all maximal orders are conjugate. It is sufficient to prove what follows: if two maximal orders, say $\Lambda$ and $\Lambda^{\prime}$ are conjugate, $\Lambda$ is right-Euclidean if and only if $\Lambda^{\prime}$ is right-Euclidean. By symmetry, suppose that $\Lambda$ is right-Euclidean with respect to $\Phi$ and let $x \in F \backslash\{0\}$ such that $\Lambda^{\prime}=x^{-1} \Lambda x$. For every $u \in \Lambda^{\prime}$ set $\Phi^{\prime}(u)=\Phi\left(x u x^{-1}\right)$. It is easy to see that $\Phi^{\prime}\left(\Lambda^{\prime}\right) \subseteq \mathbf{Z}_{\geq 0}$ and that for every $\left(a^{\prime}, b^{\prime}\right) \in \Lambda^{\prime} \times \Lambda^{\prime} \backslash\{0\}$ there exists a $q^{\prime} \in \Lambda^{\prime}$ such that $\Phi^{\prime}\left(a^{\prime}-b^{\prime} q^{\prime}\right)<\Phi^{\prime}\left(b^{\prime}\right)$. In fact, if $a^{\prime}=x^{-1} a x$ and $b^{\prime}=x^{-1} b x$ where $(a, b) \in \Lambda \times \Lambda \backslash\{0\}$, we can take $q^{\prime}=x^{-1} q x$ where $q \in \Lambda$ is such that $\Phi(a-b q)<\Phi(b)$.

This leads to

Definition 2.11. A Euclidean quaternion field is a quaternion field admitting a Euclidean order, or equivalently such that every maximal order is Euclidean.

2.4. When $\Phi$ is the norm. Let us denote by $m_{K}$ the local Euclidean minimum map of $K$ (for the norm form) defined by $m_{K}(x)=\inf _{X \in \mathbf{Z}_{K}}\left|N_{K / \mathbf{Q}}(x-X)\right|$ for $x \in K$. Let $M(K)=$ sup $m_{K}(x)$ be the Euclidean minimum of $K$. In the same way, let us introduce the notions $x \in K$ of local (and global) Euclidean minima of an order $\Lambda$ of $F$.

Definition 2.12. For any $\xi \in F$, we set

$$
m_{\Lambda}(\xi)=\inf _{\lambda \in \Lambda} N(\xi-\lambda)
$$

and we call it the local Euclidean minimum of $\Lambda$ at $\xi$. We define the Euclidean minimum of $\Lambda$ by

$$
M(\Lambda)=\sup _{\xi \in F} m_{\Lambda}(\xi)
$$


Let us notice that this supremum is a well-defined positive real number and that for every $\xi \in F$ there exists a $\lambda \in \Lambda$ such that $m_{\Lambda}(\xi)=N(\xi-\lambda)$ (see [5] and [3]).

Now, let us remark that if an order of $F$ is right norm-Euclidean, we know, by Proposition 2.6. that it is actually both left and right-Euclidean, but is it left norm-Euclidean? Looking at the proof of Proposition 2.6, we see that the answer is yes, because $N(x)=N(\bar{x})$. Using the multiplicativity of $N$, it is easy to see that we have the more precise following result.

Proposition 2.13. The following three statements are equivalent.

(i) $\Lambda$ is left-norm-Euclidean;

(ii) $\Lambda$ is right-norm-Euclidean;

(iii) For all $\xi \in F, m_{\Lambda}(\xi)<1$.

Proof. (i) or (ii) implies (iii) because every element of $F$ can be written $b^{-1} a$ or $a b^{-1}$ where $a, b \in \Lambda, b \neq 0$. Then (iii) implies (i) by considering $\xi=b^{-1} a$ which gives (2). In the same way, (iii) implies (ii) by taking $\xi=a b^{-1}$.

This allows us to speak of a norm-Euclidean order without specifying whether it is left norm-Euclidean or right norm-Euclidean. Obviously, with the above notation, if $M(\Lambda)<1$, then $\Lambda$ is norm-Euclidean. From Proposition 2.8, we know that a norm-Euclidean order is necessarily maximal, and, as in the general case, we also have:

Proposition 2.14. If $F$ admits a norm-Euclidean (necessarily maximal) order $\Lambda$, then every maximal order $\Lambda^{\prime}$ of $F$ is norm-Euclidean. Moreover, we have $M\left(\Lambda^{\prime}\right)=M(\Lambda)$.

Proof. We know that the class number $h_{F}$ is equal to 1 . As a consequence, we obtain that if $F$ admits a norm-Euclidean order $\Lambda$, every maximal order $\Lambda^{\prime}$ of $F$ is a conjugate of $\Lambda$ because $t_{F}=1$. This implies that $\Lambda^{\prime}=x^{-1} \Lambda x$ for some $x \in F \backslash\{0\}$. Then for every $\xi$ of $F$, we have trivially

$$
\begin{aligned}
m_{\Lambda^{\prime}}(\xi) & =\inf _{\lambda^{\prime} \in \Lambda^{\prime}} N\left(\xi-\lambda^{\prime}\right) \\
& =\inf _{\lambda \in \Lambda} N\left(\xi-x^{-1} \lambda x\right) \\
& =m_{\Lambda}\left(x \xi x^{-1}\right) .
\end{aligned}
$$

Since $\xi \longmapsto x \xi x^{-1}$ is a bijection of $F$, we deduce from the equality above that $\Lambda^{\prime}$ is normEuclidean and that $M\left(\Lambda^{\prime}\right)=M(\Lambda)$.

Remark 2.15. Note that the latter equality is true as soon as $t_{F}=1$. But when $t_{F}>1$, we can have two maximal orders with distinct Euclidean minima. For instance, in $F=$ $\left(\frac{-1,-11}{\mathbf{Q}}\right)$, let us consider the two maximal orders

$$
\left\{\begin{array}{l}
\Lambda=\mathbf{Z} \oplus i \mathbf{Z} \oplus \frac{i+j}{2} \mathbf{Z} \oplus \frac{1+k}{2} \mathbf{Z} \\
\Lambda^{\prime}=\mathbf{Z} \oplus \frac{1+2 i+j}{2} \mathbf{Z} \oplus 2 i \mathbf{Z} \oplus \frac{2-i-k}{4} \mathbf{Z} .
\end{array}\right.
$$

Then we have $M(\Lambda)=\frac{18}{11}$ and $M\left(\Lambda^{\prime}\right)=\frac{16}{11}$. See [5] for more details. 
Proposition 2.14 allows us to speak of norm-Euclidean quaternion fields without giving any reference to the maximal order that we consider. A norm-Euclidean quaternion field is a quaternion field admitting a norm-Euclidean order, or equivalently such that every maximal order is Euclidean. Moreover if $t_{F}=1$, in particular if $F$ is norm-Euclidean, we can speak without any ambiguity of its Euclidean minimum: $M(F)=M(\Lambda)$ for any maximal order $\Lambda$ of $F$.

Example 2.16. If $F=\left(\frac{-1,-1}{\mathbf{Q}}\right),\left(\frac{-1,-3}{\mathbf{Q}}\right)$ and $\left(\frac{-2,-5}{\mathbf{Q}}\right)$, then $M(F)$ is respectively equal to $\frac{1}{2}, \frac{2}{3}$ and $\frac{4}{5}$ (see [5]).

Let us summarize.

- If we want to prove that $F$ is norm-Euclidean, it is sufficient to choose a maximal order $\Lambda$ of $F$ and to prove that $\Lambda$ is right norm-Euclidean (or left norm-Euclidean).

- If we want to prove that $F$ is not Euclidean, we have to find a maximal order $\Lambda$ that is not right-Euclidean (or not left-Euclidean).

\section{Totally Definite QUATERnion FIEldS OVER QUADRATIC NUMBER FIELDS}

From now on, $K$ will be a real quadratic number field $K=\mathbf{Q}(\sqrt{d})$, where $d>1$ is a squarefree integer, and $F$ will be a totally definite quaternion field over $K$. As usual, we denote by $\mathbf{Z}_{K}$ the ring of integers of $K$ and by $d_{K}$ the discriminant of the field $K$. Let $\Lambda$ be a maximal order of $F$. Since we are looking for Euclidean quaternion fields, we can restrict ourselves to those with class number 1 and use the following result (see [12] or [7]).

Theorem 3.1. There are only thirteen totally definite quaternion fields $F$ over a real quadratic field with class number 1.

In Table 1, we describe these quaternion fields. The notation in the table has already been introduced, except $\mathfrak{D}$, which is the discriminant of $F$, i.e. the (squarefree) product of all finite primes ramified in $F$. When $\mathfrak{D} \neq \mathbf{Z}_{k}$ the finite primes of $K$ that ramify in $F$ are specified: as in the previous section $\mathfrak{p}_{m}$ or $\overline{\mathfrak{p}_{m}}$ (its conjugate) is a prime ideal of $\mathbf{Z}_{k}$ lying above the prime $m \in \mathbf{Z}$ and we write $\mathfrak{p}_{m}=(\omega)$ for $\mathfrak{p}_{m}=\omega \mathbf{Z}_{K}$.

In order to prove Theorem 1.5, we will first establish that four of our thirteen candidates, more precisely $F_{1}, F_{6}, F_{10}$ and $F_{13}$ are norm-Euclidean. This will be done in Subsection 3.1 . Then, in Subsection 3.2 we will prove that the nine other ones are not Euclidean.

3.1. Norm-Euclidean quaternion fields. First, recall that in [3] explicit bounds for $M(\Lambda)$ were established under a supplementary assumption on the fundamental unit of $\mathbf{Z}_{K}$.

Theorem 3.2. Let $F$ be a totally definite quaternion field over a real quadratic field $K$ and let $\Lambda$ be a maximal order of $F$. Suppose that no finite place of $K$ is ramified in $F$ and that the fundamental unit of $\mathbf{Z}_{K}$ has norm -1 . Then

$$
\frac{d_{K}}{7552+3072 \sqrt{6}} \leq M(\Lambda) \leq \frac{d_{K}}{16} .
$$

We deduce immediately from the upper bound of (5) that three of the candidates of Table 1 are norm-Euclidean.

Proposition 3.3. The quaternion fields $F_{1}, F_{6}$ and $F_{10}$ are norm-Euclidean. 


\begin{tabular}{|c|c|c|c|}
\hline$F$ & $K$ & $\mathfrak{D}$ & $(a, b)$ \\
\hline$F_{1}$ & $\mathbf{Q}(\sqrt{2})$ & $\mathbf{Z}_{K}$ & $(-1,-1)$ \\
\hline$F_{2}$ & $\mathbf{Q}(\sqrt{2})$ & $\mathfrak{p}_{2} \mathfrak{p}_{3}=(\sqrt{2})(3)$ & $(-3, \sqrt{2}-2)$ \\
\hline$F_{3}$ & $\mathbf{Q}(\sqrt{2})$ & $\mathfrak{p}_{2} \mathfrak{p}_{5}=(\sqrt{2})(5)$ & $(\sqrt{2}-2,-5)$ \\
\hline$F_{4}$ & $\mathbf{Q}(\sqrt{2})$ & $\mathfrak{p}_{2} \mathfrak{p}_{7}=(\sqrt{2})(1+2 \sqrt{2})$ & $(-\sqrt{2}-4,-1)$ \\
\hline$F_{5}$ & $\mathbf{Q}(\sqrt{2})$ & $\mathfrak{p}_{2} \overline{\mathfrak{p}_{7}}=(\sqrt{2})(1-2 \sqrt{2})$ & $(\sqrt{2}-4,-1)$ \\
\hline$F_{6}$ & $\mathbf{Q}(\sqrt{5})$ & $\mathbf{Z}_{K}$ & $(-1,-1)$ \\
\hline$F_{7}$ & $\mathbf{Q}(\sqrt{5})$ & $\mathfrak{p}_{2} \mathfrak{p}_{5}=(2)(\sqrt{5})$ & $\left(\frac{\sqrt{5}-5}{2},-2\right)$ \\
\hline$F_{8}$ & $\mathbf{Q}(\sqrt{5})$ & $\mathfrak{p}_{2} \mathfrak{p}_{11}=(2)\left(\frac{7+\sqrt{5}}{2}\right)$ & $(-1,2 \sqrt{5}-8)$ \\
\hline$F_{9}$ & $\mathbf{Q}(\sqrt{5})$ & $\mathfrak{p}_{2} \overline{\bar{p}_{11}}=(2)\left(\frac{7-\sqrt{5}}{2}\right)$ & $(-1,-2 \sqrt{5}-8)$ \\
\hline$F_{10}$ & $\mathbf{Q}(\sqrt{13})$ & $\mathbf{Z}_{K}$ & $(-1,-1)$ \\
\hline$F_{11}$ & $\mathbf{Q}(\sqrt{13})$ & $\mathfrak{p}_{2} \mathfrak{p}_{3}=(2)\left(\frac{1+\sqrt{13}}{2}\right)$ & $(-1,-2 \sqrt{13}-8)$ \\
\hline$F_{12}$ & $\mathbf{Q}(\sqrt{13})$ & $\mathfrak{p}_{2} \overline{\mathfrak{p}_{3}}=(2)\left(\frac{1-\sqrt{13}}{2}\right)$ & $(-1,2 \sqrt{13}-8)$ \\
\hline$F_{13}$ & $\mathbf{Q}(\sqrt{17})$ & $\mathbf{Z}_{K}$ & $(-1,-3)$ \\
\hline
\end{tabular}

TABLE 1 . Totally definite quaternion fields $F$ over a real quadratic field with class number 1

Proof. It is sufficient to check that in each case the fundamental unit of $K$ has norm -1 . For $F_{1}, F_{6}$ and $F_{10}$ these units are respectively $1+\sqrt{2}, \frac{1+\sqrt{5}}{2}$ and $\frac{3+\sqrt{13}}{2}$, which satisfy the condition. As the discriminants are respectively 8,5 and 13 we have in each case, that $M(\Lambda)<1$.

Let us note that Lenstra has already pointed out that these quaternion fields are normEuclidean (see [8]). Now, it remains to prove that $F_{13}$ is norm-Euclidean, which cannot be established using the previous bound since in this case $d_{K}=17>16$. Our approach will be algorithmic, following some ideas used in [4] for the computation of the Euclidean minimum of totally real number fields. Let us work in a more general context. Let $d>1$ be a squarefree integer and $F=\left(\frac{a, b}{K}\right)$ be a totally definite quaternion field over $K=\mathbf{Q}(\sqrt{d})$, where $a, b$ are supposed to belong to $\mathbf{Q}$, for simplicity. Since $F$ is totally definite, we have $a, b<0$. Let $\Lambda$ be a maximal order of $F$. Suppose that we have a description of $\Lambda$ :

$$
\Lambda=\underset{l=1}{4}\left(a_{l, 1}+a_{l, 2} i+a_{l, 3} j+a_{l, 4} k\right) \mathbf{Z}_{K},
$$

where $a_{l, m} \in K$ for $1 \leq l, m \leq 4$. Then $F$ can be written

$$
\begin{aligned}
F & =\stackrel{\oplus}{l=1}^{4}\left(a_{l, 1}+a_{l, 2} i+a_{l, 3} j+a_{l, 4} k\right) K \\
& =\Lambda \oplus \Delta,
\end{aligned}
$$

where

$$
\Delta=\underset{l=1}{\oplus}\left(a_{l, 1}+a_{l, 2} i+a_{l, 3} j+a_{l, 4} k\right) D
$$


and where $D$ is a fundamental domain of $K$. Take for instance $D=\{a+b \theta ;(a, b) \in$ $[0,1) \cap \mathbf{Q}\}$, where $\theta=\frac{1+\sqrt{d}}{2}$ if $d \equiv 1 \bmod 4$ and $\theta=\sqrt{d}$ otherwise. Now, since $m_{\Lambda}$ is $\Lambda$ periodic, to prove that $F$ is norm-Euclidean, it is sufficient to establish that for every $\xi \in \Delta$ there exists a $\lambda \in \Lambda$ such that $N(\xi-\lambda)<1$. The sets $\Lambda$ and $\Delta$ can be rewritten:

$$
\begin{gathered}
\Lambda=\left\{\sum_{l=1}^{4} a_{l, 1} z_{l}+i \sum_{l=1}^{4} a_{l, 2} z_{l}+j \sum_{l=1}^{4} a_{l, 3} z_{l}+k \sum_{l=1}^{4} a_{l, 4} z_{l} ; x_{l}, y_{l} \in \mathbf{Z}\right\}, \\
\Delta=\left\{\sum_{l=1}^{4} a_{l, 1} z_{l}+i \sum_{l=1}^{4} a_{l, 2} z_{l}+j \sum_{l=1}^{4} a_{l, 3} z_{l}+k \sum_{l=1}^{4} a_{l, 4} z_{l} ; x_{l}, y_{l} \in \mathbf{Q} \cap[0,1)\right\},
\end{gathered}
$$

where $z_{l}=x_{l}+y_{l} \theta$. Clearly, $\Lambda$ and $\Delta$ are respectively isomorphic to $\mathbf{Z}^{8}$ and $[0,1)^{8}$, and we embed both sets in $\mathbf{R}^{8}$ in the following way. Let us denote by $\sigma$ the non-trivial $\mathbf{Q}$ isomorphism of $K$ defined by $\sigma(\sqrt{d})=-\sqrt{d}$. We consider the matrix $M \in M_{8 \times 8}(\mathbf{R})$ defined by

$$
M=\left(\begin{array}{cccccccc}
a_{1,1} & a_{1,1} \theta & a_{2,1} & a_{2,1} \theta & a_{3,1} & a_{3,1} \theta & a_{4,1} & a_{4,1} \theta \\
\sigma\left(a_{1,1}\right) & \sigma\left(a_{1,1} \theta\right) & \sigma\left(a_{2,1}\right) & \sigma\left(a_{2,1} \theta\right) & \sigma\left(a_{3,1}\right) & \sigma\left(a_{3,1} \theta\right) & \sigma\left(a_{4,1}\right) & \sigma\left(a_{4,1} \theta\right) \\
a_{1,2} & a_{1,2} \theta & a_{2,2} & a_{2,2} \theta & a_{3,2} & a_{3,2} \theta & a_{4,2} & a_{4,2} \theta \\
\sigma\left(a_{1,2}\right) & \sigma\left(a_{1,2} \theta\right) & \sigma\left(a_{2,2}\right) & \sigma\left(a_{2,2} \theta\right) & \sigma\left(a_{3,2}\right) & \sigma\left(a_{3,2} \theta\right) & \sigma\left(a_{4,2}\right) & \sigma\left(a_{4,2} \theta\right) \\
a_{1,3} & a_{1,3} \theta & a_{2,3} & a_{2,3} \theta & a_{3,3} & a_{3,3} \theta & a_{4,3} & a_{4,3} \theta \\
\sigma\left(a_{1,3}\right) & \sigma\left(a_{1,3} \theta\right) & \sigma\left(a_{2,3}\right) & \sigma\left(a_{2,3} \theta\right) & \sigma\left(a_{3,3}\right) & \sigma\left(a_{3,3} \theta\right) & \sigma\left(a_{4,3}\right) & \sigma\left(a_{4,3} \theta\right) \\
a_{1,4} & a_{1,4} \theta & a_{2,4} & a_{2,4} \theta & a_{3,4} & a_{3,4} \theta & a_{4,4} & a_{4,4} \theta \\
\sigma\left(a_{1,4}\right) & \sigma\left(a_{1,4} \theta\right) & \sigma\left(a_{2,4}\right) & \sigma\left(a_{2,4} \theta\right) & \sigma\left(a_{3,4}\right) & \sigma\left(a_{3,4} \theta\right) & \sigma\left(a_{4,4}\right) & \sigma\left(a_{4,4} \theta\right)
\end{array}\right)
$$

and we see $\Lambda$ and $\Delta$ respectively as $M \cdot \mathbf{Z}^{8}$ and $M \cdot(\mathbf{Q} \cap[0,1))^{8}$. Now, as in the totally real number field case (see [4]), we consider a cutting-covering of $\bar{\Delta}=M \cdot[0,1]^{8}$ using parallelotopes whose faces are orthogonal to the canonical axes of $\mathbf{R}^{8}$. These parallelotopes $\mathcal{P}$ are of the form

$$
\mathcal{P}=\left\{\left(u_{l}\right)_{1 \leq l \leq 8} \in \mathbf{R}^{8} ;\left|u_{l}-C_{l}\right| \leq h_{l}\right\},
$$

where $C=\left(c_{l}\right)_{1 \leq l \leq 8}$ is the center of the parallelotope and $0<h_{l}$ for every $l$. In order to prove that $F$ is norm-Euclidean, it is sufficient to prove that for every $\mathcal{P}$ of our cutting-covering of $\bar{\Delta}$ there exists a $\lambda \in \Lambda$ such that

$$
\text { for every } u \in \mathcal{P}, N(u-\lambda)<1 \text {. }
$$

In this case we will say that $\mathcal{P}$ is absorbed by $\lambda$. But thanks to our identification $N$ can be rewritten

$$
N(t)=\left(t_{1}^{2}-a t_{3}^{2}-b t_{5}^{2}+a b t_{7}^{2}\right)\left(t_{2}^{2}-a t_{4}^{2}-b t_{6}^{2}+a b t_{8}^{2}\right),
$$

so that, to ensure that (6) is satisfied, it is enough to establish that

$$
A(\mathcal{P}, \lambda) \cdot B(\mathcal{P}, \lambda)<1
$$

where

$A(\mathcal{P}, \lambda)=\left(\left|C_{1}-\lambda_{1}\right|+h_{1}\right)^{2}-a\left(\left|C_{3}-\lambda_{3}\right|+h_{3}\right)^{2}-b\left(\left|C_{5}-\lambda_{5}\right|+h_{5}\right)^{2}+a b\left(\left|C_{7}-\lambda_{7}\right|+h_{7}\right)^{2}$

and

$B(\mathcal{P}, \lambda)=\left(\left|C_{2}-\lambda_{2}\right|+h_{2}\right)^{2}-a\left(\left|C_{4}-\lambda_{4}\right|+h_{4}\right)^{2}-b\left(\left|C_{6}-\lambda_{6}\right|+h_{6}\right)^{2}+a b\left(\left|C_{8}-\lambda_{8}\right|+h_{8}\right)^{2}$. 
Let us remark that this test is optimal: in fact, since $a, b<0$, for every $u \in \mathcal{P}$ we have

$$
N(u-\lambda) \leq A(\mathcal{P}, \lambda) \cdot B(\mathcal{P}, \lambda)
$$

and there exists a vertex $V$ of $\mathcal{P}$ such that

$$
N(V-\lambda)=A(\mathcal{P}, \lambda) \cdot B(\mathcal{P}, \lambda) .
$$

Now, it is sufficient to prove that every $\mathcal{P}$ of our cutting-covering satisfies (77) for some $\lambda$ belonging to a finite set $\mathcal{S}$ of precomputed elements of $\Lambda$. Of course, things are not so simple: in general, if we begin with a reasonable cutting-covering, some parallelotopes are not absorbed. In this case, we cut them into $2^{8}$ smaller parallelotopes and we continue. The algorithm is roughly as follows.

(1) Define a set $\mathcal{S}$ of elements of $\Lambda$.

(2) Define a covering of $\bar{\Delta}$ by parallelotopes as described above. Denote by $T$ the set of these parallelotopes.

(3) For any $\mathcal{P} \in T$, search for a $\lambda$ in $S$ that absorbs $\mathcal{P}$, replacing 1 by a constant $k<1$ in (77) to control rounding errors. If such a $\lambda$ exists, remove $\mathcal{P}$ from $T$.

(4) If $T=\emptyset$ we are done and the algorithm stops.

(5) If not, cut every $\mathcal{P} \in T$ into $2^{8}$ smaller parallelotopes and replace $T$ with the set of these smaller parallelotopes. Then go to step (3).

In the case of $F_{13}$ we have $K=\mathbf{Q}(\sqrt{17}), \theta=\frac{1+\sqrt{17}}{2}$ and as a maximal order for $F_{13}$ we can take

$$
\Lambda=\mathbf{Z}_{K} \oplus i \mathbf{Z}_{K} \oplus \frac{i+j}{2} \mathbf{Z}_{K} \oplus \frac{3+3 \theta i+(\theta-2) j+k}{6} \mathbf{Z}_{K},
$$

with $i^{2}=-1$ and $j^{2}=-3$. The algorithm ran with the following parameters: the set $\mathcal{S}$ was defined by $\mathcal{S}=\left\{M \cdot X ; X_{i} \in \mathbf{Z} \cap[-3,4]\right.$ for every $\left.i\right\}$, the cutting-covering of $\bar{\Delta}$ was obtained by cutting $\bar{\Delta}$ by 60 in each direction, and the constant $k$ was equal to 0.95 . After 2 loops, all parallelotopes were absorbed at one step or another and we obtained:

Proposition 3.4. The quaternion field $F_{13}$ is norm-Euclidean.

3.2. Other candidates. Now, we will prove that any maximal order of one of the remaining candidates cannot be Euclidean. We can use Corollary 2.4 in the following way. Suppose that $\Lambda$ is Euclidean, so that the sequence $\left(\Lambda_{n}\right)_{n \geq 0}$ exhausts $\Lambda$. We have trivially $\Lambda_{0}=\{0\}$ and $\Lambda_{1}=\{0\} \cup \Lambda^{\times} \neq \Lambda$. If $\Lambda_{2}=\Lambda_{1}$, then the sequence $\left(\Lambda_{n}\right)_{n \geq 0}$ is stationary from $n=1$, which is impossible. So we must have $\Lambda_{1} \subsetneq \Lambda_{2}$, and for the same reason, if $\Lambda_{2} \neq \Lambda$ we must have $\Lambda_{2} \subsetneq \Lambda_{3}$. We will prove that such a situation is impossible and that, consequently, $\Lambda$ will never be Euclidean. But before proving this fact, we need some preliminary results. Recall that, since $F$ is totally definite, $\left[\Lambda^{\times}: \mathbf{Z}_{K}^{\times}\right]$is finite. We can even specify this index.

Lemma 3.5. The index $\left[\Lambda^{\times}: \mathbf{Z}_{K}^{\times}\right]$has the following values:

- $\left[\Lambda^{\times}: \mathbf{Z}_{K}^{\times}\right]=1$ for $F=F_{3}$;

- $\left[\Lambda^{\times}: \mathbf{Z}_{K}^{\times}\right]=2$ for $F=F_{8}, F_{9}, F_{11}$ and $F_{12}$;

- $\left[\Lambda^{\times}: \mathbf{Z}_{K}^{\times}\right]=3$ for $F=F_{2}$;

- $\left[\Lambda^{\times}: \mathbf{Z}_{K}^{\times}\right]=4$ for $F=F_{4}$ and $F_{5}$;

- $\left[\Lambda^{\times}: \mathbf{Z}_{K}^{\times}\right]=5$ for $F=F_{7}$. 
Proof. As the level of $\Lambda$, which is maximal, is trivial, and as $K$ has class number 1 , the Eichler mass formula (see [12]) gives us

$$
\frac{1}{\left[\Lambda^{\times}: \mathbf{Z}_{K}^{\times}\right]}=\frac{1}{2}\left|\zeta_{K}(-1)\right| \prod_{\mathfrak{p} \mid \mathfrak{D}}(\mathrm{N}(\mathfrak{p})-1),
$$

where $\mathrm{N}(\mathfrak{p})$ is the norm of the ideal $\mathfrak{p}$ and $\zeta_{K}$ the Dedekind zeta function of $K$. Then, we can use Pari [2] to compute $\zeta_{K}(-1)$ and to conclude.

From now on we denote by $\varphi_{b}$ the canonical map $\Lambda \longrightarrow \Lambda / b \Lambda$.

Lemma 3.6. Let $b \in \Lambda \backslash\{0\}$. The map $\varphi_{b}$ has the following properties:

(i) If $\lambda \in \Lambda$, then

$$
\left|\varphi_{b}\left(\lambda \mathbf{Z}_{K}^{\times}\right)\right| \leq\left|\varphi_{b}\left(\mathbf{Z}_{K}^{\times}\right)\right|
$$

(ii) For any subset $T$ of $\Lambda$, if $a, c \in \Lambda$ satisfy $a-c \in b \Lambda$ then $\varphi_{b}(a T)=\varphi_{b}(c T)$.

Proof. (i) Let $r=\left|\varphi_{b}\left(\mathbf{Z}_{K}^{\times}\right)\right|$and let $\left\{u_{i} \in \mathbf{Z}_{K}^{\times} ; 1 \leq i \leq r\right\}$ be an exact set of representatives of $\mathbf{Z}_{K}^{\times}$modulo $b \Lambda$, i.e. such that for every $x \in \mathbf{Z}_{K}^{\times}$there exists a unique $i$ with $x-u_{i} \in b \Lambda$. If we prove that for every $y \in \lambda \mathbf{Z}_{K}^{\times}$there exists some $1 \leq i \leq r$ such that $y-\lambda u_{i} \in b \Lambda$, this will show that $\left\{\lambda u_{i} ; 1 \leq i \leq r\right\}$ is a set of representatives of $\lambda \mathbf{Z}_{K}^{\times}$modulo $b \Lambda$ (not necessarily exact), which will imply the desired inequality. Let $y=\lambda u$ with $u \in \mathbf{Z}_{K}^{\times}$. There is some $1 \leq i \leq r$ such that $u-u_{i} \in b \Lambda$ and this implies

$$
u \lambda-u_{i} \lambda=\left(u-u_{i}\right) \lambda \in b \Lambda .
$$

But $u$ and $u_{i}$, which belong to $\mathbf{Z}_{K}^{\times}$, commute with every element of $\Lambda$ and (8) leads to $y-\lambda u_{i} \in b \Lambda$.

(ii) An element of $\varphi_{b}(a T)$ is a class $a x+b \Lambda$ with $x \in T$. Since $(a-c) x \in b \Lambda$, we have $a x+b \Lambda=c x+b \Lambda \in \varphi_{b}(c T)$, so that $\varphi_{b}(a T) \subseteq \varphi_{b}(c T)$. By symmetry, we have an equality.

Now let $b \in \Lambda$ such that $b \notin \Lambda^{\times} \cup\{0\}$. The proper integral ideal $b \Lambda$ admits a decomposition into a proper product $b \Lambda=\mathfrak{N}_{1} \cdots \mathfrak{N}_{l}$ where the $\mathfrak{N}_{i}$ are maximal integral ideals. Then $\mathfrak{N}_{i} \cap \mathbf{Z}_{K}$ is a prime ideal $\mathfrak{p}_{i}$ of $\mathbf{Z}_{K}$. Let us denote by $p_{i}$ the prime below $\mathfrak{p}_{i}$ and by $f_{i}$ the residual degree of $\mathfrak{p}_{i}$.

Proposition 3.7. With this notation, let $v_{1}, \ldots, v_{s}(s \geq 1)$ be elements of $\Lambda$ such that for every $i, v_{i} \notin b \Lambda$. If

$$
\varphi_{b}\left(\{0\} \cup \bigcup_{i=1}^{s} v_{i} \Lambda^{\times}\right)=\Lambda / b \Lambda,
$$

then

$$
\prod_{i=1}^{l} p_{i}^{f_{i}}+1 \leq s\left[\Lambda^{\times}: \mathbf{Z}_{K}^{\times}\right]
$$

Proof. Let us put $m=\left[\Lambda^{\times}: \mathbf{Z}_{K}^{\times}\right]$and let $\left\{t_{j} ; 1 \leq j \leq m\right\}$ be an exact set of representatives of $\Lambda^{\times}$modulo $\mathbf{Z}_{K}^{\times}$so that $\Lambda^{\times}=\cup_{j=1}^{m} t_{j} \mathbf{Z}_{K}^{\times}$. For every $i$, we have

$$
\left|\varphi_{b}\left(v_{i} \Lambda^{\times}\right)\right|=\left|\bigcup_{j=1}^{m} \varphi_{b}\left(v_{i} t_{j} \mathbf{Z}_{K}^{\times}\right)\right|,
$$


from which we deduce by Lemma 3.6 (i)

$$
\left|\varphi_{b}\left(v_{i} \Lambda^{\times}\right)\right| \leq m\left|\varphi_{b}\left(\mathbf{Z}_{K}^{\times}\right)\right| .
$$

Now, lets us put $r=\left|\varphi_{b}\left(\mathbf{Z}_{K}^{\times}\right)\right|$and, as above, let $\left\{u_{i} \in \mathbf{Z}_{K}^{\times} ; 1 \leq i \leq r\right\}$ be an exact set of representatives of $\mathbf{Z}_{K}^{\times}$modulo $b \Lambda$. The sets $u_{i}+\mathfrak{p}_{1} \cdots \mathfrak{p}_{l}$ are disjoint: if not, there would exist some $i \neq j$ with

$$
u_{i}-u_{j} \in \mathfrak{p}_{1} \cdots \mathfrak{p}_{l} \subseteq \mathfrak{N}_{1} \cdots \mathfrak{N}_{l}=b \Lambda,
$$

which is impossible by choice of the $u_{i}$. Moreover, for every $i, u_{i} \notin \mathfrak{p}_{1} \cdots \mathfrak{p}_{l}$ because $u_{i} \in \mathbf{Z}_{K}^{\times}$. So, the $u_{i}+\mathfrak{p}_{1} \cdots \mathfrak{p}_{l}$ can be viewed as distinct non trivial elements of $\mathbf{Z}_{K} / \mathfrak{p}_{1} \cdots \mathfrak{p}_{l}$ and we obtain

$$
r \leq\left[\mathbf{Z}_{K}: \mathfrak{p}_{1} \cdots \mathfrak{p}_{l}\right]-1=\prod_{j=1}^{l} p_{j}^{f_{j}}-1 .
$$

From (10) and (11) we obtain that for every $1 \leq i \leq s,\left|\varphi_{b}\left(v_{i} \Lambda^{\times}\right)\right| \leq m\left(\prod_{j=1}^{l} p_{j}^{f_{j}}-1\right)$, and consequently that

$$
\left|\varphi_{b}\left(\bigcup_{i=1}^{s} v_{i} \Lambda^{\times}\right)\right| \leq s m\left(\prod_{j=1}^{l} p_{j}^{f_{j}}-1\right) .
$$

We also know that for every integral ideal $I$ with right order $\Lambda,|\Lambda / I|=N_{K / \mathbf{Q}}\left(\operatorname{nrd}_{F / K}(I)\right)^{2}$, so that

$$
|\Lambda / b \Lambda|=N_{K / \mathbf{Q}}\left(\operatorname{nrd}_{F / K}\left(\mathfrak{N}_{1} \cdots \mathfrak{N}_{l}\right)\right)^{2} .
$$

By Lemma 2.2 (i) we have

$$
\operatorname{nrd}_{F / K}\left(\mathfrak{N}_{1} \cdots \mathfrak{N}_{l}\right)=\operatorname{nrd}_{F / K}\left(\mathfrak{N}_{1}\right) \cdots \operatorname{nrd}_{F / K}\left(\mathfrak{N}_{l}\right),
$$

from which we deduce

$$
|\Lambda / b \Lambda|=N_{K / \mathbf{Q}}\left(\operatorname{nrd}_{F / K}\left(\mathfrak{N}_{1}\right)\right)^{2} \cdots N_{K / \mathbf{Q}}\left(\operatorname{nrd}_{F / K}\left(\mathfrak{N}_{l}\right)\right)^{2} .
$$

As $\operatorname{nrd}_{F / K}\left(\mathfrak{N}_{i}\right)=\mathfrak{p}_{i}$ and $N_{K / \mathbf{Q}}\left(\mathfrak{p}_{i}\right)=p_{i}^{f_{i}}$ we finally obtain

$$
|\Lambda / b \Lambda|=\prod_{i=1}^{l} p_{i}^{2 f_{i}}
$$

By hypothesis, we have $\varphi_{b}\left(\{0\} \cup \bigcup_{i=1}^{s} v_{i} \Lambda^{\times}\right)=\Lambda / b \Lambda$, which implies

$$
\left|\varphi_{b}\left(\bigcup_{i=1}^{s} v_{i} \Lambda^{\times}\right)\right|=|\Lambda / b \Lambda|-1,
$$

because for every $i, v_{i} \notin b \Lambda$ so that $\bigcup_{i=1}^{s} v_{i} \Lambda^{\times} \cap b \Lambda=\emptyset$. From (13) we have

$$
\left|\varphi_{b}\left(\bigcup_{i=1}^{s} v_{i} \Lambda^{\times}\right)\right|=\prod_{i=1}^{l} p_{i}^{2 f_{i}}-1 .
$$

Finally (12) and (14) give

$$
\prod_{i=1}^{l} p_{i}^{2 f_{i}}-1 \leq s m\left(\prod_{j=1}^{l} p_{j}^{f_{j}}-1\right),
$$


which leads to the result.

Corollary 3.8. The quaternion fields $F_{i}$, for $i \in\{2,3,4,5,7,8,9,11,12\}$, are not Euclidean.

Proof. We suppose that $\Lambda$ is Euclidean. We have seen that this implies $\Lambda_{1}=\{0\} \cup \Lambda^{\times} \subsetneq \Lambda_{2}$ and that, if $\Lambda_{2} \neq \Lambda, \Lambda_{2} \subsetneq \Lambda_{3}$. We consider four cases.

Case 1: $i \in\{3,8,9,11,12\}$.

As we must have $\Lambda_{1} \subsetneq \Lambda_{2}$, there exists some $b \notin\{0\} \cup \Lambda^{\times}$such that

$$
\varphi_{b}\left(\{0\} \cup \Lambda^{\times}\right)=\Lambda / b \Lambda .
$$

Of course, as $b \notin \Lambda^{\times}, 1 \notin b \Lambda$ and Proposition 3.7 applied with $s=1$ and $v_{1}=1$ leads to

$$
\prod_{i=1}^{l} p_{i}^{f_{i}}+1 \leq\left[\Lambda^{\times}: \mathbf{Z}_{K}^{\times}\right]
$$

where the notation is the same as above. But $\prod_{i=1}^{l} p_{i}^{f_{i}}+1 \geq 3$ and in all cases, $\left[\Lambda^{\times}: \mathbf{Z}_{K}^{\times}\right] \leq 2$. This is a contradiction.

Case 2: $i \in\{4,5\}$. As before, we see that there must be some $b \notin\{0\} \cup \Lambda^{\times}$such that $\varphi_{b}\left(\{0\} \cup \Lambda^{\times}\right)=\Lambda / b \Lambda$. In both cases $\left[\Lambda^{\times}: \mathbf{Z}_{K}^{\times}\right]=4$ and Proposition 3.7 shows that, necessarily, $l=1$, so that $b \Lambda=\mathfrak{N}$ is a maximal integral ideal. Moreover if $\mathfrak{p}=\mathfrak{N} \cap \mathbf{Z}_{K}$ and if $p$ is the prime below $\mathfrak{p}$, Proposition 3.7 implies $p^{f} \leq 3$ where $f$ is the residual degree of $\mathfrak{p}$. Since 3 is inert in $K=\mathbf{Q}(\sqrt{2})$, the only possibility is $p=2$ and $\mathfrak{p}=\sqrt{2} \mathbf{Z}_{K}$.

In the case $F=F_{4}$, a maximal order of $F$ is

$$
\Lambda=\mathbf{Z}_{K} \oplus i \mathbf{Z}_{K} \oplus \frac{1+\sqrt{2}+\sqrt{2} i+j}{2} \mathbf{Z}_{K} \oplus \frac{(\sqrt{2}+1) i+k}{2} \mathbf{Z}_{K},
$$

where $i^{2}=-\sqrt{2}-4$ and $j^{2}=-1$. We can compute $\Lambda^{\times} / \mathbf{Z}_{K}^{\times}$and find that its 4 elements are the following classes:

But

$$
\mathbf{Z}_{K}^{\times}, j \mathbf{Z}_{K}^{\times},-\frac{\sqrt{2}}{2}(1+j) \mathbf{Z}_{K}^{\times} \text {and } \frac{\sqrt{2}}{2}(1-j) \mathbf{Z}_{K}^{\times} .
$$

$$
j-1=\sqrt{2}\left(\sqrt{2} \frac{1+\sqrt{2}+\sqrt{2} i+j}{2}-i-1-\sqrt{2}\right) \in \sqrt{2} \Lambda \subseteq \mathfrak{N}=b \Lambda
$$

and

$$
\frac{\sqrt{2}}{2}(1+j)+\frac{\sqrt{2}}{2}(1-j)=\sqrt{2} \in \sqrt{2} \Lambda \subseteq \mathfrak{N}=b \Lambda .
$$

By Lemma 3.6 (ii) these relations imply that

$$
\varphi_{b}\left(\Lambda^{\times}\right) \subseteq \varphi_{b}\left(\mathbf{Z}_{K}^{\times}\right) \bigcup \varphi_{b}\left(\frac{\sqrt{2}}{2}(1-j) \mathbf{Z}_{K}^{\times}\right)
$$

so that, by Lemma 3.6 (i), we have

$$
\left|\varphi_{b}\left(\Lambda^{\times}\right)\right| \leq 2\left|\varphi_{b}\left(\mathbf{Z}_{K}^{\times}\right)\right| .
$$

But (11) indicates that $\left|\varphi_{b}\left(\mathbf{Z}_{K}^{\times}\right)\right| \leq 2^{1}-1=1$ and finally we obtain $\left|\varphi_{b}\left(\Lambda^{\times}\right)\right| \leq 2$. On the one hand, this implies

$$
\left|\varphi_{b}\left(\{0\} \cup \Lambda^{\times}\right)\right| \leq 3
$$


And, on the other hand, (13) gives us

$$
|\Lambda / b \Lambda|=p^{2 f}=4,
$$

which contradicts $\varphi_{b}\left(\{0\} \cup \Lambda^{\times}\right)=\Lambda / b \Lambda$.

The proof is the same for $F=F_{5}$.

Case 3: $i=2$. In this case $\left[\Lambda^{\times}: \mathbf{Z}_{K}^{\times}\right]=3$. Once again, there must be some $b \notin\{0\} \cup \Lambda^{\times}$ such that $\varphi_{b}\left(\{0\} \cup \Lambda^{\times}\right)=\Lambda / b \Lambda$, and by Proposition 3.7 we find: $l=1, b \Lambda=\mathfrak{N}$ is a maximal integral ideal and $\mathfrak{p}=\mathfrak{N} \cap \mathbf{Z}_{K}$ is the prime ideal $\sqrt{2} \mathbf{Z}_{K}$ lying above 2. Moreover by Lemma 2.2 (ii), $\mathfrak{N}$ is the unique maximal ideal containing $\sqrt{2} \mathbf{Z}_{K}$. Now, if $c \notin\{0\} \cup \Lambda^{\times}$is such that $\varphi_{c}\left(\{0\} \cup \Lambda^{\times}\right)=\Lambda / c \Lambda$, we have $c \Lambda=\mathfrak{N}=b \Lambda$ and $c \in b \Lambda^{\times}$. Conversely, if $c \in b \Lambda^{\times}$, we have $c \Lambda=b \Lambda, \varphi_{c}=\varphi_{b}$ and $\varphi_{c}\left(\{0\} \cup \Lambda^{\times}\right)=\Lambda / c \Lambda$. This implies that

$$
\Lambda_{2}=\{0\} \cup \Lambda^{\times} \cup b \Lambda^{\times} .
$$

Since $21 \Lambda_{2} \neq \Lambda$, we have $\Lambda_{2} \subsetneq \Lambda_{3}$ and there exists some $d \in \Lambda$ such that

$$
d \notin\{0\} \cup \Lambda^{\times} \cup b \Lambda^{\times}
$$

and

$$
\varphi_{d}\left(\{0\} \cup \Lambda^{\times} \cup b \Lambda^{\times}\right)=\Lambda / d \Lambda .
$$

Obviously, (15) implies that $1 \notin d \Lambda$. Suppose that $b \in d \Lambda$. Then $b \Lambda \subseteq d \Lambda$, and by maximality of $b \Lambda=\mathfrak{N}$, we have either $d \Lambda=\Lambda$ or $d \Lambda=b \Lambda$. This implies either $d \in \Lambda^{\times}$or $d \in b \Lambda^{\times}$. This is a contradiction. Consequently, $1, b \notin d \Lambda$ and we can apply Proposition [3.7 with $s=2$, $v_{1}=1$ and $v_{2}=b$. With the above notation, we obtain

$$
\prod_{i=1}^{l} p_{i}^{f_{i}}+1 \leq 6 .
$$

But 3 and 5 are inert in $K=\mathbf{Q}(\sqrt{2})$ and the only possibility is again: $l=1, p_{1}=2$. By uniqueness of $\mathfrak{N}$, this implies $d \Lambda=\mathfrak{N}=b \Lambda$ and $d \in b \Lambda^{\times}$, which is absurd.

Case 4: $i=7$. To treat this case, we need to specify $\Lambda$. As a maximal order of $F_{7}$ we can take

$$
\Lambda=\mathbf{Z}_{K} \oplus \frac{\frac{\sqrt{5}+1}{2}+i}{2} \mathbf{Z}_{K} \oplus j \mathbf{Z}_{K} \oplus \frac{\frac{\sqrt{5}+1}{2} j+k}{2} \mathbf{Z}_{K}
$$

with $i^{2}=(\sqrt{5}-5) / 2$ and $j^{2}=-2$. Here $\left[\Lambda^{\times}: \mathbf{Z}_{K}^{\times}\right]=5$ and we can precise $\Lambda^{\times} / \mathbf{Z}_{K}^{\times}$. Its five elements are the classes $\alpha_{i} \mathbf{Z}_{K}^{\times}$, where the $\alpha_{i}(1 \leq i \leq 5)$ are respectively

$$
1, \frac{-\sqrt{5}+1-(\sqrt{5}+1) i}{4}, \frac{\sqrt{5}-1-(\sqrt{5}+1) i}{4}, \frac{-\sqrt{5}-1+2 i}{4}, \frac{\sqrt{5}+1+2 i}{4},
$$

so that we have

$$
\Lambda^{\times}=\bigcup_{i=1}^{5} \alpha_{i} \mathbf{z}_{K}^{\times} \text {and } j \Lambda^{\times}=\bigcup_{i=1}^{5} j \alpha_{i} \mathbf{Z}_{K}^{\times}
$$

As before, there must exist some $b \notin\{0\} \cup \Lambda^{\times}$such that $\varphi_{b}\left(\{0\} \cup \Lambda^{\times}\right)=\Lambda / b \Lambda$. Since 3 is inert in $\mathbf{Z}_{K}$, Proposition 3.7 gives: $l=1, b \Lambda=\mathfrak{N}$ where $\mathfrak{N}$ is the unique maximal integral ideal above the prime ideal $2 \mathbf{Z}_{K}$, by Lemma 2.2 (ii). But $j \in \Lambda$ satisfies $\operatorname{nrd}_{F / K}(j)=2$, and

\footnotetext{
${ }^{2}$ If not, $N_{K / \mathbf{Q}}\left(\operatorname{nrd}_{F / K}(\Lambda)\right)$ would be finite but contains $N_{K / \mathbf{Q}}^{2}\left(\mathbf{Z}_{K}\right)$.
} 
by Lemma 2.2 (iv) we have $j \Lambda=\mathfrak{N}=b \Lambda$. Using the same argument as before, we finally obtain

$$
\Lambda_{2}=\{0\} \cup \Lambda^{\times} \cup j \Lambda^{\times} .
$$

Again $\Lambda_{2} \subsetneq \Lambda$ and there exists some $d \in \Lambda$ such that

$$
d \notin\{0\} \cup \Lambda^{\times} \cup j \Lambda^{\times}
$$

and

$$
\varphi_{d}\left(\{0\} \cup \Lambda^{\times} \cup j \Lambda^{\times}\right)=\Lambda / d \Lambda .
$$

As in case 3 , we see that $1, j \notin d \Lambda$ and we can apply Proposition 3.7 with $s=2, v_{1}=1$ and $v_{2}=j$. We obtain

$$
\prod_{i=1}^{l} p_{i}^{f_{i}}+1 \leq 10
$$

and the only possibilities are $l=1$ and $p_{1}=2\left(\right.$ with $\left.f_{1}=2\right), l=1$ and $p_{1}=3\left(\right.$ with $\left.f_{1}=2\right)$ or $l=1$ and $p_{1}=5$ (with $f_{1}=1$ ). Let us analyze the three cases.

- Subcase 1. By uniqueness of $\mathfrak{N}=j \Lambda$, this case leads, as before, to a contradiction.

- Subcase 2. We have $d \Lambda=\mathfrak{N}^{\prime}$ where $\mathfrak{N}^{\prime}$ is a (not necessarily unique) maximal ideal such that $\mathfrak{N}^{\prime} \cap \mathbf{Z}_{K}=3 \mathbf{Z}_{K}$. As it will be useful later, let us remark that we can check from the values of the $\alpha_{i}$ or from the equality $j \Lambda=\Lambda j$, that we have

$$
j \Lambda^{\times}=\Lambda^{\times} j \text {. }
$$

As $\varphi_{d}\left(\Lambda_{2}\right)=\Lambda / d \Lambda$, there exists some $\lambda_{2} \in \Lambda_{2}$ such that $j+1+d \Lambda=\lambda_{2}+d \Lambda$. Then $\lambda_{2}=j+1-d \lambda$ for some $\lambda \in \Lambda$. But $\lambda_{2} \in \Lambda_{2}$ so that either $\lambda_{2}=0$, either $\lambda_{2} \in \Lambda^{\times}$or $\lambda_{2} \in j \Lambda^{\times}$. We claim that in the three cases, there exists some $\varepsilon \in \Lambda^{\times}$such that

$$
j-\varepsilon \in d \Lambda \text {. }
$$

- In the first case, $\lambda_{2}=0$ implies $j+1=d \lambda$ and we can take $\varepsilon=-1$.

- Let us analyze the second case where $\lambda_{2} \in \Lambda^{\times}$. We have $\lambda_{2}(j-1)=-3-d \lambda(j-1)$ and, as $3 \in \mathfrak{N}^{\prime}=d \Lambda$ and $j-1 \in \Lambda$,

$$
\lambda_{2}(j-1) \in d \Lambda .
$$

But $\lambda_{2}(j-1)=\lambda_{2} j-\lambda_{2}=j \alpha-\lambda_{2}$ for some $\alpha \in \Lambda^{\times}$by (17). This leads to $\lambda_{2}(j-1) \alpha^{-1}=$ $j-\lambda_{2} \alpha^{-1}$ and from (19) we obtain

$$
j-\lambda_{2} \alpha^{-1} \in d \Lambda
$$

Here we can take $\varepsilon=\lambda_{2} \alpha^{-1} \in \Lambda^{\times}$.

- Now, if $\lambda_{2} \in j \Lambda^{\times}$, by (17) there exist $\alpha, \beta \in \Lambda^{\times}$such that $\lambda_{2}=j \alpha=\beta j$. As before $\lambda_{2}(j-1)=-3-d \lambda(j-1) \in d \Lambda$. But $\lambda_{2}(j-1)=\beta j(j-1)=-2 \beta-\beta j=-3 \beta+\beta-j \alpha$ which implies $j \alpha-\beta=-\lambda_{2}(j-1)-3 \beta \in d \Lambda$. Thus we have

$$
j-\beta \alpha^{-1} \in d \Lambda
$$

and we can take $\varepsilon=\beta \alpha^{-1}$.

Our claim is proved. From (18), we see by Lemma [3.6 (ii) that $\varphi_{d}\left(j \Lambda^{\times}\right)=\varphi_{d}\left(\varepsilon \Lambda^{\times}\right)=$ $\varphi_{d}\left(\Lambda^{\times}\right)$. Finally

$$
\Lambda / d \Lambda=\varphi_{d}\left(\Lambda_{2}\right)=\varphi_{d}\left(\Lambda_{1}\right)
$$

which implies $d \in \Lambda_{2}$. This is a contradiction. 
- Subcase 3. Since the prime ideal $\sqrt{5} \mathbf{Z}_{K}$ is ramified in $F$, we have $d \Lambda=\mathfrak{N}^{\prime}$ where $\mathfrak{N}^{\prime}$ is the unique maximal (and two-sided) ideal such that $\mathfrak{N}^{\prime} \cap \mathbf{Z}_{K}=\sqrt{5} \mathbf{Z}_{K}$. An easy computation shows that $\operatorname{nrd}_{F / K}\left(\alpha_{3}-\alpha_{1}\right)=\sqrt{5} \frac{\sqrt{5}-1}{2} \in \sqrt{5} \mathbf{Z}_{K}$ and $\operatorname{nrd}_{F / K}\left(\alpha_{4}-\alpha_{1}\right)=\operatorname{nrd}_{F / K}\left(\alpha_{5}-\alpha_{2}\right)=$ $\sqrt{5} \frac{\sqrt{5}+1}{2} \in \sqrt{5} \mathbf{Z}_{K}$. By Lemma 2.2 (iv) this implies $\alpha_{3}-\alpha_{1}, \alpha_{4}-\alpha_{1}, \alpha_{5}-\alpha_{2} \in \mathfrak{N}^{\prime}=d \Lambda$, and, because $\mathfrak{N}^{\prime}$ is two-sided, that $j \alpha_{3}-j \alpha_{1}, j \alpha_{4}-j \alpha_{1}, j \alpha_{5}-j \alpha_{2} \in \mathfrak{N}^{\prime}=d \Lambda$. Then, using Lemma 3.6 (ii), we can write

$$
\varphi_{d}\left(\{0\} \cup \Lambda^{\times} \cup j \Lambda^{\times}\right)=\varphi_{d}(\{0\}) \cup \bigcup_{i=1}^{2} \varphi_{d}\left(\alpha_{i} \mathbf{Z}_{K}^{\times}\right) \cup \bigcup_{i=1}^{2} \varphi_{d}\left(j \alpha_{i} \mathbf{Z}_{K}^{\times}\right),
$$

and Lemma 3.6 (i) together with (11) leads to

$$
\left|\varphi_{d}\left(\{0\} \cup \Lambda^{\times} \cup j \Lambda^{\times}\right)\right| \leq 1+4\left|\varphi_{d}\left(\mathbf{Z}_{K}^{\times}\right)\right| \leq 1+4 \cdot\left(5^{1}-1\right)=17 .
$$

But we must have $\left|\varphi_{d}\left(\{0\} \cup \Lambda^{\times} \cup j \Lambda^{\times}\right)\right|=|\Lambda / d \Lambda|$ and the latter cardinality is

$$
\left|\Lambda / \mathfrak{N}^{\prime}\right|=N_{K / \mathbf{Q}}\left(\operatorname{nrd}_{F / K}\left(\mathfrak{N}^{\prime}\right)\right)^{2}=N_{K / \mathbf{Q}}\left(\sqrt{5} \mathbf{Z}_{K}\right)^{2}=25 .
$$

This is a contradiction.

Finally, Proposition 3.3, Proposition 3.4 and Corollary 3.8 give us Theorem 1.1.

Remark 3.9. Note that the situation is the same as in the imaginary quadratic fields case: Euclideanity and norm-Euclideanity are equivalent. Moreover we have examples of quaternion fields with class number one, that are not Euclidean.

\section{Concluding Remark}

Our main purpose was to study Euclidean totally definite quaternion fields over quadratic fields. Incidently, we have discovered that this question was not yet solved for (totally) definite quaternion fields over $\mathbf{Q}$. As already mentionned, we know that $\left(\frac{-1,-1}{\mathbf{Q}}\right),\left(\frac{-1,-3}{\mathbf{Q}}\right)$ and $\left(\frac{-2,-5}{\mathbf{Q}}\right)$ are the only norm-Euclidean definite quadratic fields over $\mathbf{Q}$ and we are naturally led to ask ourselves whether there are other such fields which are Euclidean although not norm-Euclidean. If we are looking for a quaternion field $F$ with this property, we must have $h_{F}=1$ and we know (see [12] or [7]) that necessarily $F=\left(\frac{-1,-7}{\mathbf{Q}}\right)$ or $\left(\frac{-2,-13}{\mathbf{Q}}\right)$. Let us write respectively $\mathcal{F}_{1}$ and $\mathcal{F}_{2}$ for these two candidates. Then we have

Proposition 4.1. Neither $\mathcal{F}_{1}$ nor $\mathcal{F}_{2}$ is Euclidean.

Proof. We use the same technique as before. Let $\Lambda$ be a maximal order of $F=\mathcal{F}_{1}$ or $\mathcal{F}_{2}$. We have $\Lambda_{0}=\{0\}, \Lambda_{1}=\{0\} \cup \Lambda^{\times}$and we will prove that $\Lambda_{1} \subsetneq \Lambda_{2}$ is impossible. A careful reading of the proof of Proposition 3.7 shows that it can be rephrased, in this context, with $s=1$ and $v_{1}=1$, more simply, in the following way. Let $b \in \Lambda_{2} \backslash \Lambda_{1}$. Then the proper integral ideal $b \Lambda$ admits a decomposition into a proper product $\mathfrak{N}_{1} \cdots \mathfrak{N}_{l}$ of maximal ideals with $l \geq 1$. Let $p_{i} \in \mathbf{Z}$ be the prime such that $\mathfrak{N}_{i} \cap \mathbf{Z}=p_{i} \mathbf{Z}$. Then we have

$$
\prod_{i=1}^{l} p_{i}+1 \leq\left[\Lambda^{\times}:\{-1,1\}\right] \text {. }
$$


Now, in this case, the Eichler mass formula is

$$
\frac{1}{\left[\Lambda^{\times}:\{-1,1\}\right]}=|\zeta(-1)| \prod_{p \mid D}(p-1),
$$

where $\zeta$ is the Riemann zeta function and $D$ is the discriminant of $F$, i.e. $D=7$ for $\mathcal{F}_{1}$ and $D=13$ for $\mathcal{F}_{2}$. Since $\zeta(-1)=-1 / 12$, we obtain $\left[\Lambda^{\times}:\{-1,1\}\right]=2$ for $\mathcal{F}_{1}$ and 1 for $\mathcal{F}_{2}$. This contradicts (20).

As a corollary of Proposition 4.1, we obtain Theorem 1.6.

\section{ACKNOWLEDGEMENTS}

The authors would like to thank Dr. Elizabeth Strouse for her stylistic advice and the anonymous referee for his suggestions of improvement.

\section{REFERENCES}

[1] Magma, v2.18-5, Sydney, 2012, http://magma.maths.usyd.edu.au/magma/

[2] PARI/GP, version 2.5.1, Bordeaux, 2012, http://pari.math.u-bordeaux.fr

[3] E. Bayer, J.-P. Cerri, J. Chaubert, Euclidean minima and central division algebras, International Journal of Number Theory 5 (2009), 1155-1168

[4] J.-P. CERri, Euclidean minima of totally real number fields: Algorithmic determination, Mathematics of Computation 76 (2007), 1547-1575

[5] J. Chaubert, Minimum euclidien des ordres maximaux dans les algèbres centrales à division, $\mathrm{PhD}$ Thesis, EPFL (2006)

[6] M. Deuring, Algebren, Springer Verlag, New-York (1968)

[7] M. Kirschmer, J. Voight, Algorithmic enumeration of ideal classes for quaternion orders, SIAM J. Comput. 39 (2010), No 5, 1714-1747

[8] H.-W. Lenstra Jr., Quelques exemples d'anneaux euclidiens, C.R. Acad. Sc. Paris, Sér. I Math. 286 (1978), 683-685

[9] T. Motzkin, On the Euclidean Algorithm, Bull. Amer. Math. Soc., 55 (1949), 1142-1146

[10] I. Reiner, Maximal orders, Claderon Press, Oxford, 2003

[11] P. SAmuel, About Euclidean Rings, Journal of Algebra, 19 (1971), 282-301

[12] M.-F. Vignéras, Arithmétique des algèbres de quaternions, Lecture Notes in Math. 800, Springer, Berlin, 1980

Univ. Bordeaux, imB, UMR 5251, F-33400 Talence, France, CNRS, imB, UMR 5251, F-33400

Talence, France, InRIA, LFAnT, F-33400 Talence, France

E-mail address: jean-paul.cerri@math.u-bordeaux1.fr

Rue du talent, 1042 Malapalud, Suisse

E-mail address: jerome.chaubert@gmail.com

Univ. Bordeaux, IMB, UMR 5251, F-33400 Talence, France, CNRS, IMB, UMR 5251, F-33400

Talence, France, INRIA, LFANT, F-33400 TAlence, France

E-mail address: pierre.lezowski@math.u-bordeaux1.fr 